\title{
Editorial
}

\section{Advanced Mesh-Based and Particle-Based Numerical Methods for Engineering and Applied Mathematics Problems}

\author{
Cheng-Tang Wu, ${ }^{1}$ Lihua Wang, ${ }^{2}$ Bernard Bonello, ${ }^{3}$ Leevan Ling, \\ Ninshu Ma, ${ }^{5}$ and Marc A. Schweitzer ${ }^{6,7}$ \\ ${ }^{1}$ Livermore Software Technology Corporation (LSTC), Livermore, CA 94551, USA \\ ${ }^{2}$ School of Aerospace Engineering and Applied Mechanics, Tongji University, Shanghai 200092, China \\ ${ }^{3}$ Institut des NanoSciences de Paris, Université Pierre et Marie Curie, 75005 Paris, France \\ ${ }^{4}$ Department of Mathematics, Hong Kong Baptist University, Kowloon Tong, Hong Kong \\ ${ }^{5}$ Center of Computational Welding Science, Osaka University, Osaka 5650871, Japan \\ ${ }^{6}$ Institute for Numerical Simulation, University of Bonn, 53115 Bonn, Germany \\ ${ }^{7}$ Fraunhofer Institute for Algorithms and Scientific Computing SCAI, 53754 Sankt Augustin, Germany \\ Correspondence should be addressed to Cheng-Tang Wu; ctwu@lstc.com
}

Received 22 February 2017; Accepted 22 February 2017; Published 19 March 2017

Copyright (C) 2017 Cheng-Tang Wu et al. This is an open access article distributed under the Creative Commons Attribution License, which permits unrestricted use, distribution, and reproduction in any medium, provided the original work is properly cited.

Thanks for the rapid progress of computing resources, many advanced numerical methods have undergone extensive developments and led to widespread applications in interdisciplinary sciences and engineering. The title of this special issue is a topic that significant research efforts have been spent on over the past decade. The development of powerful mesh-based and particle-based numerical methods for both routine and sophisticated engineering problems with complete solutions has been recognized by most researchers to benefit the industry as a whole, while creating new avenues for further research and spearheading of pioneering efforts within the field of computation and industrial applications. The objective of this special issue is to present the state-ofthe-art and prospective directions of those advanced numerical methods from the research areas to computation and application fields for engineering and applied mathematical problems.

This special issue contains 12 peer-reviewed papers. Different from the use of traditional computational methods, L. Huang et al. consider the Lattice-Boltzmann method for a flow simulation of suspension bridge cable. F. Qin et al. conduct a geological anomaly design using an extreme value theory. W. Duan et al. study the internal wave dynamic problems using the low model analysis. X. Shi et al. predict the rate of penetration in drilling problems using an efficient machine learning approach. Y. Liu and K. Din propose a numerical method based on Daubechies wavelet basis and Bspline patches for elasticity problems. J. Zhao et al. present an improved ant colony algorithm for solving the path planning problem for omnidirectional mobile vehicle. X. B. Gu and Q. H. Wu apply the nonordinary state-based peridynamic theory for the damage process of rock materials. J. Cai et al. apply the discrete element method for the analysis of large-diameter cast-in-place concrete piles. C. Liu et al. developed a multisensor scheduling algorithm based on the hybrid task decomposition and a modified binary particle swarm optimization. A new numerical method based on cubic spline function is developed by M. Hu and F. Li for solving the nonlinear dynamics problems. A new multiobjective algorithm based the quantum-behaved particle swarm optimization is proposed by D. Zhou et al. Finally, a meshless radial basis function based on the partition of unity method is developed by $\mathrm{S}$. Li et al. for the analysis of piezoelectric structures.

\section{Acknowledgments}

We would like to thank all who kindly contributed their papers to this special issue. We are also indebted to the 
reviewers and publishing team for their assistance during the preparation and publication of this special issue.

Cheng-Tang $W u$ Lihua Wang

Bernard Bonello

Leevan Ling

Ninshu Ma

Marc A. Schweitzer 


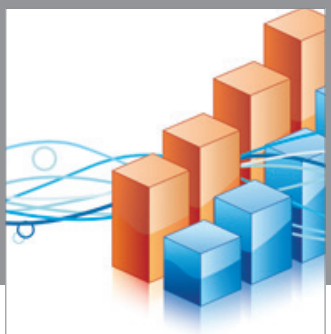

Advances in

Operations Research

vatem alat4

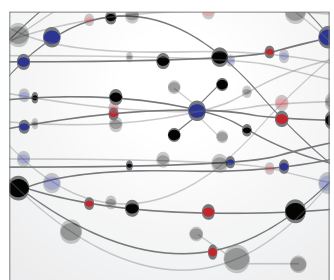

\section{The Scientific} World Journal
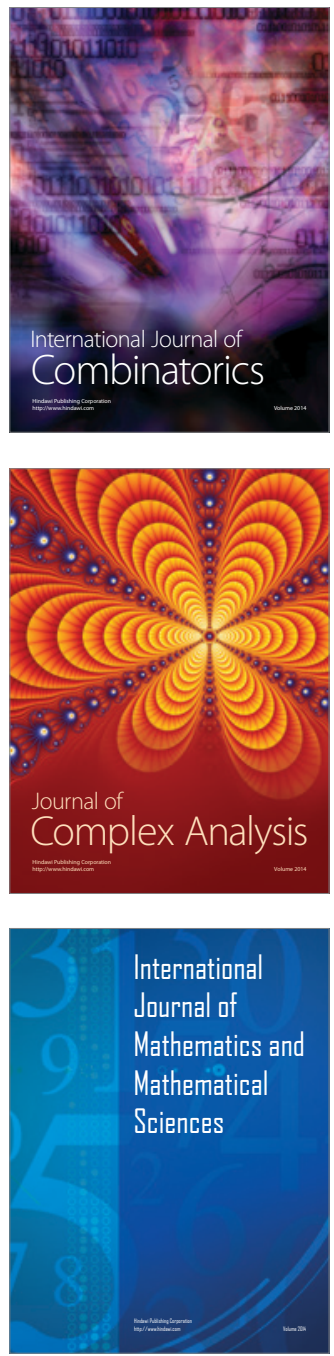
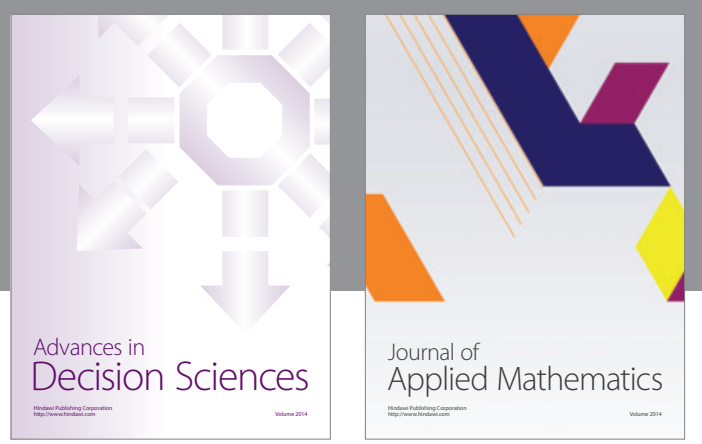

Algebra

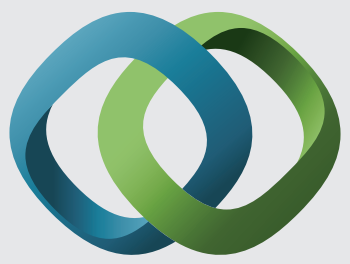

\section{Hindawi}

Submit your manuscripts at

https://www.hindawi.com
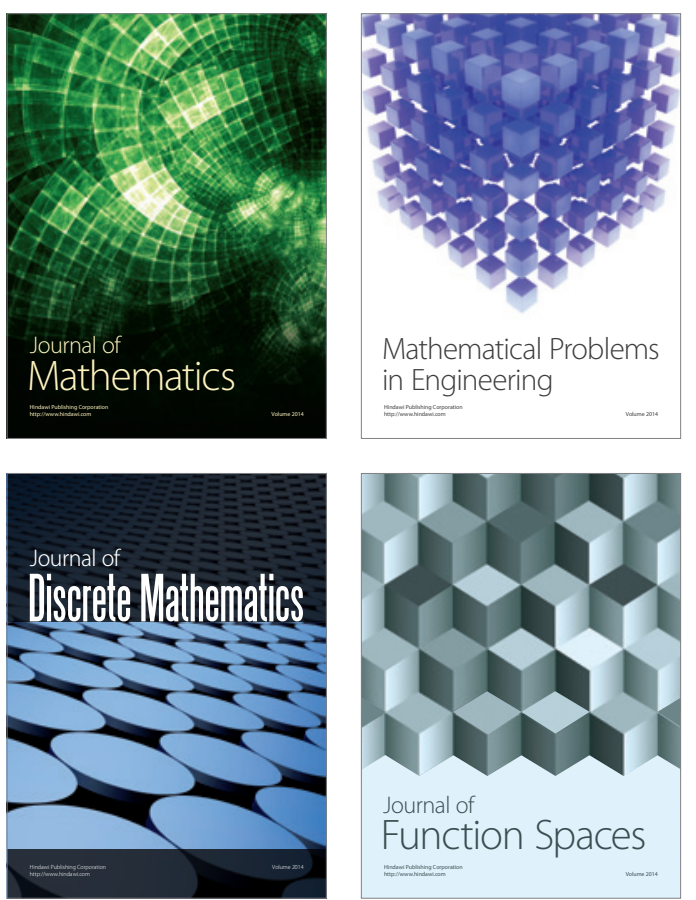

Mathematical Problems in Engineering
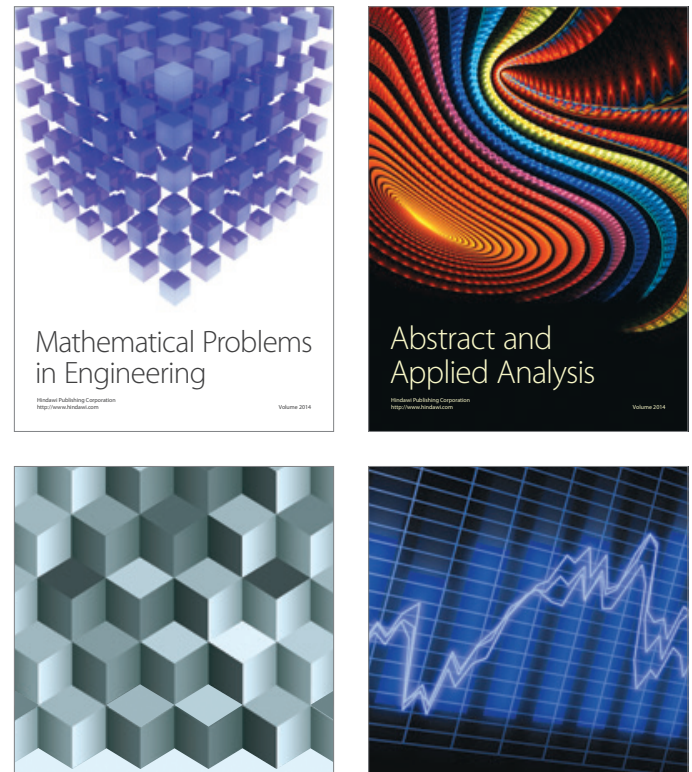

Journal of

Function Spaces

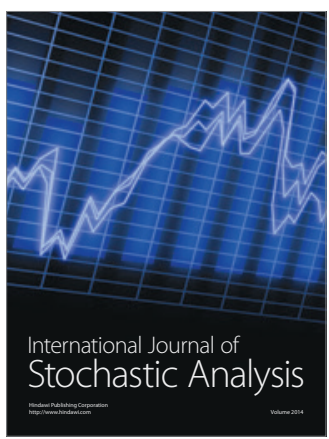

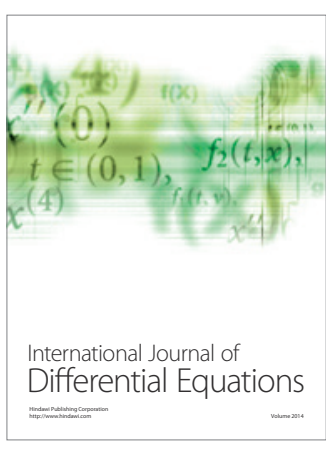
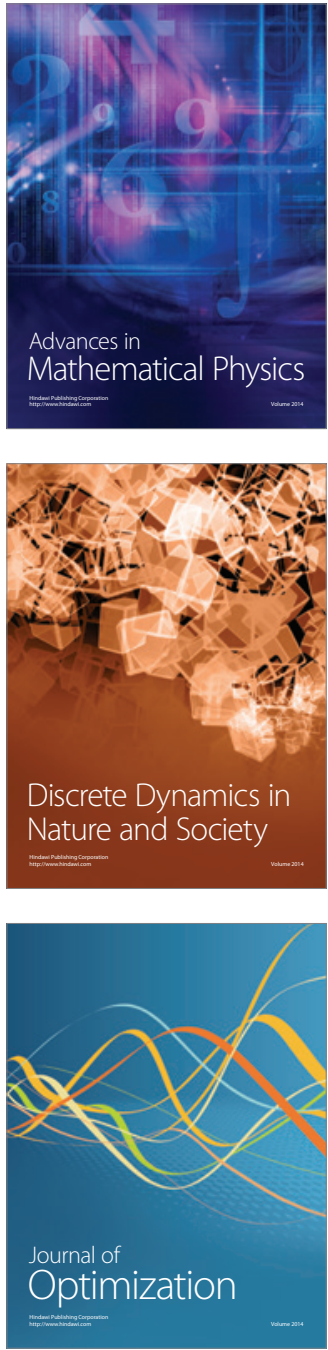